\title{
A note on d'Alembert's functional equation on a restricted domain
}

\author{
AnNA BAHYRYCZ AND JANusz BRZDȨK
}

\begin{abstract}
Let $A$ be a subgroup of a commutative group $(G,+)$ and $P$ be a quadratically closed field. We give the full description of all pairs of functions $f: G \rightarrow P$ and $g: A \rightarrow P$ such that $f(x+y)+f(x-y)=2 f(x) g(y)$ for $(x, y) \in G \times A$.
\end{abstract}

Mathematics Subject Classification (2010). 39B52, 39B55.

Keywords. d'Alembert's functional equation, cosine function, restricted domain, quadratically closed field.

In [4] we gave a full description of functions $f$ mapping a commutative group $(G,+)$ into a quadratically closed field $P$ and satisfying the condition

$$
f(x+y)+f(x-y)=2 f(x) f(y)
$$

for all $(x, y) \in A \times G$, where $A$ is a subgroup of $G$. With this note we would like to complement those results and provide a description of functions $f$ fulfilling (1) for all $(x, y) \in G \times A$; those two issues seem to be very similar, but actually they differ significantly (see Remark 1). Next, it is natural to generalize the problem and consider it for Wilson's first generalization of (1)

$$
f(x+y)+f(x-y)=2 f(x) g(y),
$$

that is to investigate functions $f: G \rightarrow P$ and $g: A \rightarrow P$ such that (2) holds for $(x, y) \in G \times A$. Note yet that, with $g(x) \equiv 1$, equation (2) becomes Jensen's equation, which is much better known in its equivalent (under suitable assumptions) form $f\left(\frac{1}{2}(x+y)\right)=\frac{1}{2}(f(x)+f(y))$. For further information and some recent results concerning the equations mentioned above see [1-9].

Throughout this paper $(G,+)$ is a commutative group, $A$ is a subgroup of $G$, and $P$ is a field that is quadratically closed (i.e., for each $a \in P$ there is $b \in P$ with $a=b^{2}$ ). If char $P \neq 2$, then for a function $m: G \rightarrow P, m_{e}$ and $m_{o}$ denote the even and odd parts of $m$, respectively, i.e., $m_{e}(x)=\frac{1}{2}(m(x)+m(-x))$ and $m_{o}(x)=\frac{1}{2}(m(x)-m(-x))$ for $x \in G$. Moreover, for $a \in G$ and $D \subset G$ we write 
$2 D:=\{2 x: x \in D\}, a+D:=\{a+b: b \in D\}$, and $a-D:=\{a-b: b \in D\}$. We need the following result from [1, Lemma 3$]$.

Theorem 1. Let char $P \neq 2$. Functions $f, g: G \rightarrow P, f(G) \neq\{0\}$, satisfy (2) for all $x, y \in G$ if and only if one of the following two statements holds.

(i) There are an exponential $m: G \rightarrow\{-1,1\}$ (i.e., $m(x+y)=m(x) m(y)$ for $x, y \in G), \alpha \in P$ and an additive $L: G \rightarrow P$ (i.e., $L(x+y)=L(x)+L(y)$ for $x, y \in G)$ with $f(x)=m(x)(L(x)+\alpha)$ and $g(x)=m(x)$ for $x \in G$.

(ii) There are an exponential $m: G \rightarrow P \backslash\{0\}$ and constants $\beta, \gamma \in P$ such that $f(x)=\beta m_{e}(x)+\gamma m_{o}(x)$ and $g(x)=m_{e}(x)$ for $x \in G$.

In what follows, if $S$ is a subgroup of $G$, then we say that a function $\xi$, mapping the factor group $G / S$ into $G$, is a lifting provided $\xi([y]) \in[y]$ for $y \in G$, where $[y]:=y+S$. We begin with the case when char $P \neq 2$.

Theorem 2. Functions $f: G \rightarrow P, f(G) \neq\{0\}$, and $g: A \rightarrow P$ satisfy (2) for $(x, y) \in G \times A$ if and only if one of the following two statements is valid.

$(\alpha)$ There are an exponential $\mu: A \rightarrow\{-1,1\}$, a family of additive functions $L_{\sigma}: A \rightarrow P$ for $\sigma \in G / A$, a lifting $\xi: G / A \rightarrow G$ with $\xi([0])=0$, and a function $\alpha: G / A \rightarrow P$ with $f(w)=\mu(w-\xi([w]))\left(L_{[w]}(w-\xi([w]))+\alpha([w])\right)$ for $w \in G$ and $g=\mu$.

( $\beta$ ) There exist an exponential $m: A \rightarrow P \backslash\{0\}$, a lifting $\xi: G / A \rightarrow G$ with $\xi([0])=0$, and functions $\beta, \gamma: G / A \rightarrow P$ such that $g=m_{e}$ and $f(w)=\beta([w]) m_{e}(w-\xi([w]))+\gamma([w]) m_{o}(w-\xi([w]))$ for $w \in G$.

Proof. Let $f, g$ satisfy (2) for $(x, y) \in G \times A$. Then, with $y=0$ in (2), we obtain $g(0)=1$. Next, replacing $y$ with $-y$ in (2) we get $2 f(x) g(-y)=f(x-$ $y)+f(x+y)=2 f(x) g(y)$ for $(x, y) \in G \times A$, which means that $g$ is even. Write $h_{x}(a)=f(x+a)$ for $(x, a) \in G \times A$. Then

$$
h_{x}(b+a)+h_{x}(b-a)=2 h_{x}(b) g(a), \quad a, b \in A, x \in G .
$$

Let $\xi: G / A \rightarrow G$ be a lifting with $\xi([0])=0$ and $H_{s}:=h_{\xi(s)}$ for $s \in G / A$. Then

$$
f(w)=f(\xi([w])+w-\xi([w]))=H_{[w]}(w-\xi([w])), \quad w \in G .
$$

Moreover, by $(3), H_{s}(b+a)+H_{s}(b-a)=2 H_{s}(b) g(a)$ for $a, b \in A, s \in G / A$.

If $g(A) \subset\{-1,1\}$, then Theorem 1 , with $f=H_{s}$, yields $g=\mu$ and $H_{s}(a)=$ $\mu(a)\left(L_{s}(a)+\alpha(s)\right)$ for $a \in A, s \in G / A$, with some family of additive functions $L_{s}: A \rightarrow P$ (for $s \in G / A$ ), an exponential $\mu: A \rightarrow\{-1,1\}$ and some $\alpha: G / A \rightarrow P$. Hence and from (4) we obtain the form of $f$ described in $(\alpha)$.

If there is $a \in A$ with $g(a) \neq 0$ and $g(a)^{2} \neq 1$, then by Theorem 1(ii), for each $s \in G / A$, there exist an exponential $m^{s}: A \rightarrow P$ and $\beta_{1}(s), \gamma_{1}(s) \in P$ such that $g=m^{s}{ }_{e}$ and $H_{s}(a)=\beta_{1}(s) m^{s}{ }_{e}(a)+\gamma_{1}(s) m^{s}{ }_{o}(a)$ for $a \in A, s \in G / A$. Clearly, with $m:=m^{[0]}$, we have

$$
m^{s}(a) \in\{m(a), m(-a)\}, \quad a \in A, s \in G / A .
$$


Suppose that there are $s \in G / A$ and $a, b \in A$ with $m^{s}(a)=m(a) \neq m(-a)$ and $m^{s}(b)=m(-b) \neq m(b)$. Then $m^{s}(a+b)=m^{s}(a) m^{s}(b) \neq m(a) m(b)=$ $m(a+b)$ and $m^{s}(a+b)=m^{s}(a) m^{s}(b) \neq m(-a) m(-b)=m(-a-b)$, which contradicts (5). Thus we have proved that $m^{s}=m$ or $m^{s}=m \circ \iota$ for $s \in G / A$, where $\iota: A \rightarrow A$ is given by: $\iota(a)=-a$ for $a \in A$. Note yet that $(m \circ \iota)_{o}=-m_{o}$. Whence and from $(4), f(w)=\beta([w]) m_{e}(w-\xi([w]))+\gamma([w]) m_{o}(w-\xi([w]))$ for $w \in G$, where $\beta(s)=\beta_{1}(s)$ and $\gamma(s)=-\gamma_{1}(s)$ if $m^{s}=m \circ \iota$ and $\gamma(s)=\gamma_{1}(s)$ if $m^{s}=m$. This completes the proof of the necessary condition.

Now we show the sufficient condition. Write $f_{s}(a):=f(\xi(s)+a)$ for $a \in$ $A, s \in G / A$. Then, from Theorem 1, we obtain that $f_{s}(a+b)+f_{s}(a-b)=$ $2 f_{s}(a) g(b)$ for $a, b \in A$. Hence $f(x+y)+f(x-y)=f_{[x]}(x-\xi([x])+y)+f_{[x]}(x-$ $\xi([x])-y)=2 f(\xi([x])+x-\xi([x])) g(y)=2 f(x) g(y)$ for $x \in G, y \in A$.

Theorem 2 (with $f=g$ ) yields at once the following.

Corollary 1. A function $f: G \rightarrow P, f(G) \neq\{0\}$, fulfils (1) for $(x, y) \in G \times A$ if and only if either

$$
f(w)=\mu(w-\xi([w]))\left(L_{[w]}(w-\xi([w]))+\alpha([w])\right), \quad w \in G
$$

with an exponential $\mu: A \rightarrow\{-1,1\}$, a family of additive functions $L_{\sigma}: A \rightarrow P$ for $\sigma \in G / A$, a lifting $\xi: G / A \rightarrow G$, and a function $\alpha: G / A \rightarrow P$ such that $\xi([0])=0, \alpha([0])=1$ and $L_{[0]}(y)=0$ for $y \in A$, or

$$
f(w)=\beta([w]) m_{e}(w-\xi([w]))+\gamma([w]) m_{o}(w-\xi([w])), \quad w \in G
$$

with an exponential $m: A \rightarrow P$, a lifting $\xi: G / A \rightarrow G$, and some functions $\beta, \gamma: G / A \rightarrow P$ such that $\xi([0])=0, \beta([0])=1$, and $\gamma([0])=0$.

Remark 1. Next, let $A \neq G, f: G \rightarrow P, f(A)=\{0\}, f(G \backslash A)=\{1\}$, and $g: A \rightarrow P, g(A)=\{1\}$. Then (2) holds for all $(x, y) \in G \times A$. But $g$ cannot be extended to a function $g_{0}: G \rightarrow P$ such that $f(x+y)+f(x-y)=2 f(x) g_{0}(y)$ for all $(x, y) \in G^{2}$, because $f(0+w)+f(0-w)=2 \neq 0=2 f(0) g(w)$ for $w \in G \backslash A$.

The situation when a pair of functions $f, g: G \rightarrow P$ satisfies (2) for all $(x, y) \in A \times G$ appears to be significantly different from that considered in this paper and it seems that the methods used here cannot be applied for it, except the case $f=g$, which can be easily reduced to the case when (1) holds for all $(x, y) \in G \times A$, because then $f$ must be even (it is enough to take $x=0$ in (1)). This and Corollary 1 yield a description of the necessary form of all $f: G \rightarrow P$ satisfying (1) for $(x, y) \in A \times G$. However, a reasonably simple description of the sufficient form of all such $f$ seems to be a nontrivial task (as we see in [4]) and therefore the results in [4] cannot be easily derived from Corollary 1.

We end the paper with the case char $P=2$, when (2) takes the form

$$
f(x+y)+f(x-y)=0 .
$$


Theorem 3. Let char $P=2$ and $B \subset G, B \neq \emptyset$. Then $f: G \rightarrow P$ satisfies (8) for $(x, y) \in G \times B$ if and only if there is $\gamma: G / S_{0} \rightarrow P$ with $f(x)=\gamma([x])$ for $x \in G$, where $S_{0}$ denotes the subgroup of $G$ that is generated by the set $2 B$.

Proof. Let $f$ satisfy (8) for all $(x, y) \in G \times B$ and $A_{0}:=\{y \in G: f(x+y)=$ $f(x)$ for $x \in G\}$. Then, for all $y, z \in A_{0}, x \in G, f(x+y-z)=f(x+y-z+z)=$ $f(x+y)=f(x)$, whence $y-z \in A_{0}$. So, $A_{0}$ is a subgroup of $G$.

Let $S_{0}$ denote the subgroup of $G$ that is generated by $2 B$. Taking $y \in B$ and replacing $x$ with $x+y$ in (8) we get $f(x+2 y)+f(x)=0$, whence $f(x+2 y)=$ $-f(x)=f(x)$ for $x \in G, y \in B$. This implies that $2 B \subset A_{0}$, whence $S_{0} \subset A_{0}$. Consequently $f(x+y)=f(x)$ for $x \in G, y \in S_{0}$. So, we may define a function $\gamma: G / S_{0} \rightarrow P$ by $\gamma([x])=f(x)$ for $x \in G$.

To complete the proof, suppose that there is $\gamma: G / S_{0} \rightarrow P$ with $f(x)=$ $\gamma([x])$ for $x \in G$. Take $x \in G$ and $y \in B$. Then $x+y-(x-y)=2 y \in 2 B \subset S_{0}$, which means that $[x+y]=[x-y]$ and consequently $f(x+y)+f(x-y)=$ $\gamma([x+y])+\gamma([x-y])=2 \gamma([x+y])=0$.

Proposition 1. Let char $P=2$ and $B \subset G, B \neq \emptyset$. Then every function $f: G \rightarrow P$ fulfilling (8) for $(x, y) \in G \times B$ satisfies (8) for $(x, y) \in G^{2}$ if and only if the subgroup of $G$ that is generated by $2 B$ is equal to $2 G$.

Proof. Let $S_{0}$ denote the subgroup generated by $2 B$. First assume that $S_{0}=$ $2 G$. Let $f: G \rightarrow P$ fulfil (8) for $(x, y) \in G \times B$. Then the function $f$ has the form described in Theorem 3. Since, for every $x, y \in G$, we have $x+y-(x-$ $y)=2 y \in 2 G=S_{0}$, this means that $f(x+y)=f(x-y)$ and consequently $f(x+y)+f(x-y)=2 f(x+y)=0$.

Now, suppose that there is $z \in 2 G \backslash S_{0}$. Then $z=2 v$ for some $v \in G$. Take $p \in P$ with $p \neq 0$ and define $\gamma: G / S_{0} \rightarrow P$ by $\gamma([z])=p$ and $\gamma([x])=0$ for $x \in G$ with $[x] \neq[z]$. Let $f(x)=\gamma([x])$ for $x \in G$. Then, by Theorem 3, (8) holds for $(x, y) \in G \times B$, but $f(v+v)+f(v-v)=f(2 v)+f(0)=p \neq 0$.

Open Access. This article is distributed under the terms of the Creative Commons Attribution License which permits any use, distribution, and reproduction in any medium, provided the original author(s) and the source are credited.

\section{References}

[1] Aczél, J., Chung, J.K., Ng, C.T.: Symmetric second differences in product form on groups. In: Rassias, Th.M. (ed.) Topics in Mathematical Analysis. Ser. Pure Math, vol. 11, pp. 1-22. World Scientific Publ. Co., Teaneck

[2] Aczél, J., Dhombres, J.: Functional Equations in Several Variables. Cambridge University Press, Cambridge (1989)

[3] D’Alembert, J.: Mémoire sur les principes de mécanique. Hist. Acad. Sci., Hist. Acad. Sci., Paris 1769, 278-286

[4] Bahyrycz, A., Brzdȩk, J.: On solutions of the d'Alembert equation on a restricted domain. Aequationes Math. 85(1-2), 169-183 (2013) 
[5] Davison, T.M.K.: D'Alembert's functional equation on topological groups. Aequationes Math. 76, 33-53 (2008)

[6] Davison, T.M.K.: D'Alembert's functional equation on topological monoids. Publ. Math. Debrecen 75, 41-66 (2009)

[7] Stetkær, H.: On operator-valued spherical functions. J. Funct. Anal. 224, 338-351 (2005)

[8] Stetkær, H.: Properties of d'Alembert functions. Aequationes Math. 77, 281-301 (2009)

[9] Yang, D.: Cosine functions revisited. Banach J. Math. Anal. 5, 126-130 (2011)

Anna Bahyrycz, Janusz Brzdȩk

Department of Mathematics

Pedagogical University

Podchorążych 2

30-084 Kraków

Poland

e-mail: bah@up.krakow.pl jbrzdek@up.krakow.pl

Received: March 1, 2013

Revised: November 11, 2013 